\title{
SENSITIVITY ANALYSIS OF TRANSIENT BIOHEAT TRANSFER DURING THERMAL INJURY FORMATION OF BIOLOGICAL TISSUE
}

\author{
Marek Jasiński \\ Institute of Computational Mechanics and Engineering \\ Silesian University of Technology, Gliwice, Poland \\ marek.jasinski@polsl.pl
}

\begin{abstract}
The sensitivity analysis of the transient temperature field in the 2D tissue domain with respect to its thermophysical parameters is discussed. In particular, the influence of tissue specific heat, thermal conductivity, perfusion rate and metabolic heat source on the temperature distribution is considered. In order to determine the influence of variations of these parameters on temperature distribution, the direct approach of sensitivity analysis is applied. The algorithm of modeling of tissue injury withdrawal based on Arrhenius integral is also presented. At the stage of numerical realization the boundary element method is used. In the final part of paper the results obtained are shown.
\end{abstract}

Keywords: bioheat transfer, tissue injury integral, tissue injury, sensitivity analysis

\section{Introduction}

During the interaction between biological tissue and external, high-temperature impulse elevated temperature and tissue damage can dynamically alter thermophysical properties of the tissue. Such processes are usually modeled by the so-called Arrhenius injury integral, in which the reaction rate increases exponentially with the temperature [1-4].

According to the Arrhenius formula, the damage of the tissue is irreversible. It follows that even in the case of a very small increase and lowering of temperature the tissue remains damaged. As a matter of fact, in the case of moderate temperature $\left(37\right.$ to $45 \div 55^{\circ} \mathrm{C}$ ) the only response of the tissue is dilatation of the blood vessels, without any thermal injury. Therefore, one can conclude that if the tissue destruction does not exceed some threshold value, the tissue has the ability to return to its native state. In the current paper the possibility of tissue damage withdrawal is taken into consideration by using of algorithm proposed in [4].

The course of the physical process is, as a rule, analyzed on the basis of a certain mathematical model. One of the problems connected with the application of such a model is the sensitivity of the solution with respect to the parameters appearing in the governing equations. The sensitivity information may be used, 
among others, to analyze the influence of the change of parameters on the final solution of the problem being considered [5-8]. Additional tasks required to determine the sensitivity functions result from differentiation of the assumed equation describing bioheat transfer with respect to the parameter, which means that the number of additional sensitivity tasks corresponds to the number of parameters with respect to which the sensitivity analysis is performed $[5,9]$.

In this paper the tissue is regarded as a homogeneous domain with a perfusion coefficient dependent on tissue necrosis, while the remaining thermal parameters are regarded as constant values. So, the sensitivity analysis has been done with respect to thermal conductivity, volumetric specific heat, an initial perfusion rate, and a metabolic heat source.

The basic problems, but also the additional problems resulting from the sensitivity analysis, have been solved using the $1^{\text {st }}$ scheme of boundary element method for transient heat diffusion $[5,10]$.

\section{Governing equations}

The transient heat transfer in the 2D homogeneous domain of biological tissue (Fig. 1) is described by the bioheat transfer equation in Pennes formulation [2]

$$
\mathbf{x} \in \Omega: \quad c \dot{T}=\lambda T_{, i i}+c_{B} G_{B}\left(T_{B}-T\right)+Q_{m e t}
$$

where $\lambda\left[\mathrm{Wm}^{-1} \mathrm{~K}^{-1}\right]$ is the thermal conductivity, $c\left[\mathrm{Jm}^{-3} \mathrm{~K}^{-1}\right]$ is the volumetric specific heat, $G_{B}\left[\left(\mathrm{~m}^{3}{ }_{\text {blood }} / \mathrm{s}\right) /\left(\mathrm{m}^{3}\right.\right.$ tissue $\left.)\right], c_{B}\left[\mathrm{Jm}^{-3} \mathrm{~K}^{-1}\right]$ and $T_{B}$ correspond to the perfusion coefficient, the volumetric specific heat of blood and the artery temperature respectively, $Q_{m e t}\left[\mathrm{Wm}^{-3}\right]$ is the internal metabolic heat source while $T=T(\mathbf{x}, t)$ and $\dot{T}$ denotes a temperature and its time derivative $[4,6,8]$.

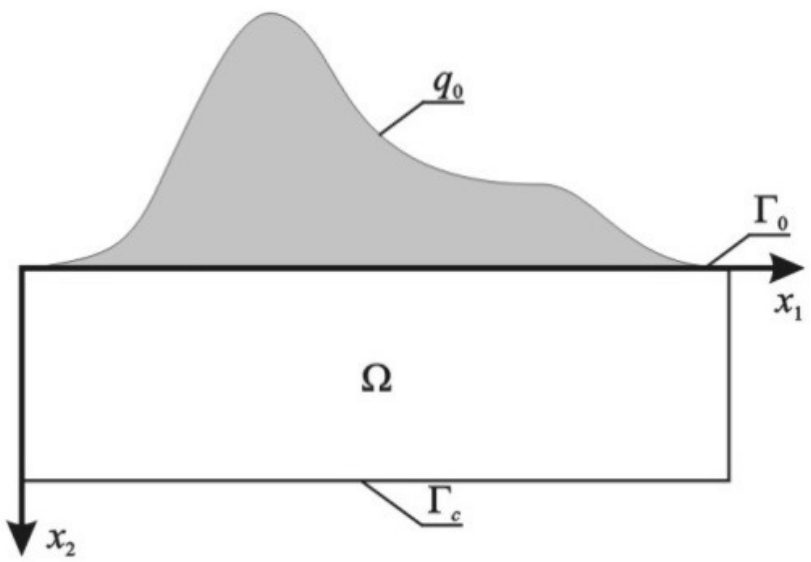

Fig. 1. Domain considered 
Equation (1) is supplemented by boundary condition along the external boundary of the tissue $\Gamma_{0}$ in the form:

$$
\mathbf{x} \in \Gamma_{0}: \begin{cases}q(\mathbf{x}, t)=q_{0}, & t \leq t_{\exp } \\ q(\mathbf{x}, t)=\alpha\left(T-T_{\text {amb }}\right), & t>t_{\text {exp }}\end{cases}
$$

where $q_{0}\left[\mathrm{Wm}^{-2}\right]$ is the known boundary heat flux, $\alpha\left[\mathrm{Wm}^{-2} \mathrm{~K}^{-1}\right]$ is the convective heat transfer coefficient and $T_{a m b}$ is the temperature of surroundings, while $t_{\exp }$ is the exposure time. On the remaining parts of the boundary $\Gamma_{c}$ the non-flux condition is accepted, and the initial distribution of temperature is also known.

In order to perform the conditions of non-controlled case of high temperature biological tissue interaction, the heat flux along boundary $\Gamma_{0}$ is assumed to be the irregular one. The distribution of the heat flux is visible in Figure 1 and it is described by the polynomial function of $7^{\text {th }}$ degree [4].

According to the necrotic changes in tissue, the blood perfusion coefficient is defined as [2]

$$
G_{B}=G_{B}(\theta)=G_{B 0} f(\theta)
$$

where $G_{B 0}$ denotes the initial perfusion coefficient while $f(\theta)$ denotes the polynomial function in the form

$$
f(\theta)=\sum_{j=1}^{3} m_{j} \theta^{j-1}
$$

where $m_{j}$ are the coefficients and $\theta$ is the Arrhenius injury integral $[2,3,6]$ :

$$
\theta(\mathbf{x})=\int_{0}^{t^{F}} A \exp \left[-\frac{\Delta E}{R T}\right] \mathrm{d} t
$$

In equation (5) $A$ is the pre-exponential factor $\left[\mathrm{s}^{-1}\right], \Delta E$ is the activation energy $\left[\mathrm{J}\right.$ mole $\left.{ }^{-1}\right]$ and $R$ is the universal gas constant $\left[\mathrm{J} \mathrm{mole}^{-1} \mathrm{~K}^{-1}\right],\left[0, t^{F}\right]$ is the considered time interval, while the criterion for tissue necrosis is $[2,3]$ :

$$
\theta(x) \geq 1
$$

The assumption of the tissue injury integral is that the damage of the tissue is irreversible. In order to consider that the tissue could get back to its native state after the thermal impulse is ceased, the following algorithm is proposed (Fig. 2).

Let us assume that for the time interval $\left[0, t^{F}\right]$ being under consideration and divided into $F$ subintervals $\left[t^{f-1}, t^{f}\right]$ (where $\left.f=1,2 \ldots F\right)$, the values $T^{0}(\mathbf{x}) \ldots T^{f}(\mathbf{x})$ as well as $\theta^{0}(\mathbf{x}) \ldots \theta^{f-1}(\mathbf{x})$ at the point $\mathbf{x} \in \Omega$ are known. At the same time the recovery threshold $\theta_{\text {rec }}$ is accepted. 
If the injury integral at the point $\mathbf{x}$ for time $t^{f}$ achieves the value equal to or greater than $\theta_{\text {rec }}$ then the injury of the tissue becomes irreversible. Otherwise the function denoted as $\theta_{a p p}(\mathbf{x}, T)$ is introduced in order to model the withdrawal of the tissue injury. In current paper it is assumed as the linear one between $\left(T^{0}, \theta^{0}\right)$ and $\left(T^{f-1}, \theta^{f-1}\right)$.

$$
\theta_{a p p}(\mathbf{x}, T)=b_{1}+b_{2} T
$$

For the time $\mathrm{t}^{f+1}$, if $T^{f+1}<T^{f}$ and $\theta^{f}<\theta_{\text {rec }}$ again the function $\theta_{a p p}$ defined for time $t^{f}$ is used [4].

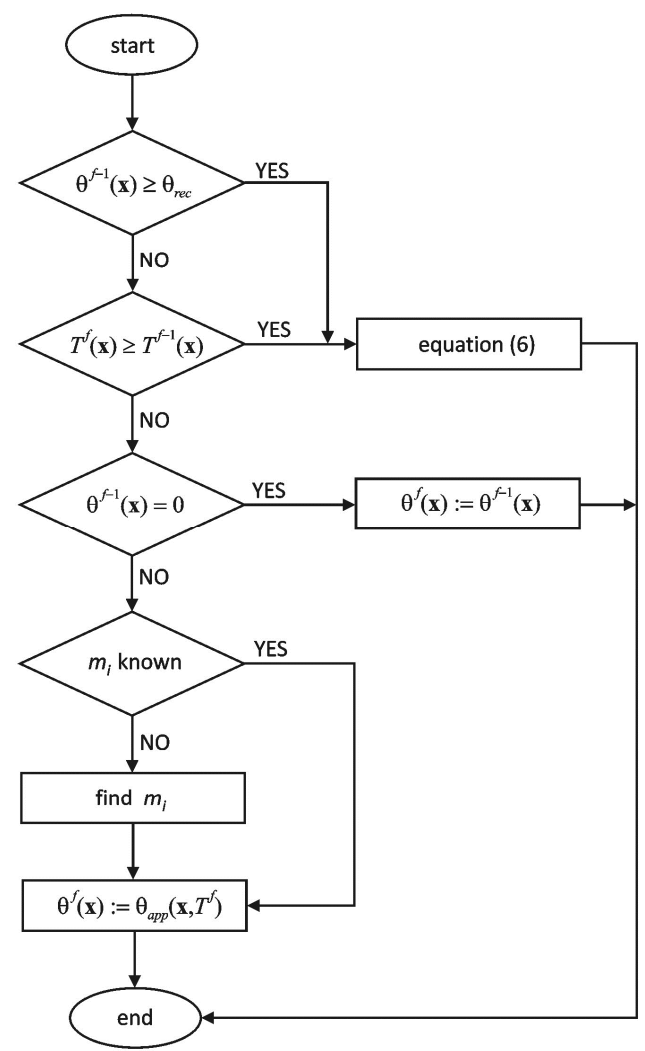

Fig. 2. The algorithm of tissue injury calculation

\section{Sensitivity analysis}

In order to determine the influence of thermophysical parameters on the temperature distribution in tissue domain, the direct approach of sensitivity analysis has been applied [7, 9]. 
According to the rules of direct method equation (1) is differentiated with respect to thermophysical parameter $p_{s}$, where $s=\lambda, c, G_{B 0}$ or $Q_{\text {met }}[5,6]$

$$
\frac{\partial c}{\partial p_{s}} \dot{T}+c \frac{\partial \dot{T}}{\partial p_{s}}=\frac{\partial \lambda}{\partial p_{s}} T_{, i i}+\lambda \frac{\partial T_{, i i}}{\partial p_{s}}+\frac{\partial}{\partial p_{s}}\left[c_{B} G_{B 0} f(\theta)\left(T_{B}-T\right)\right]+\frac{\partial Q_{m e t}}{\partial p_{s}}
$$

After the mathematical manipulations one can write the equation for an additional sensitivity problem as

$$
\mathbf{x} \in \Omega: \quad c \dot{U}^{s}=\lambda U_{, i i}^{s}+Q_{V}^{s}
$$

where

$$
U^{s}=\frac{\partial T}{\partial p_{s}}
$$

denotes the sensitivity function of the parameter $p_{s}$, while [7]

$$
\dot{U}^{s}=\frac{\partial U^{s}}{\partial t}, \quad U_{, i i}^{s}=\frac{\partial T_{, i i}}{\partial p_{s}}
$$

The source component $Q_{V}^{s}$ of the equation (9) is in the form

$$
\begin{aligned}
& Q_{V}^{s}=\left[\frac{k}{\lambda} \frac{\partial \lambda}{\partial p_{s}}-\frac{k}{G_{B 0}} \frac{\partial G_{B 0}}{\partial p_{s}}-\frac{k}{f(\theta)} \frac{\partial f(\theta)}{\partial p_{s}}\right]\left(T-T_{B}\right) \\
& -k U^{s}+\left(\frac{c}{\lambda} \frac{\partial \lambda}{\partial p_{s}}-\frac{\partial c}{\partial p_{s}}\right) \dot{T}-\frac{Q_{m e t}}{\lambda} \frac{\partial \lambda}{\partial p_{s}}+\frac{\partial Q_{\text {met }}}{\partial p_{s}}
\end{aligned}
$$

where

$$
k=c_{B} G_{B 0} f(\theta)
$$

and the derivative function $f(\theta)$ with respect to parameter $p_{s}$ is as follows

$$
\frac{\partial f(\theta)}{\partial p_{s}}=m_{2} \frac{\partial \theta}{\partial p_{s}}+2 m_{3} \theta \frac{\partial \theta}{\partial p_{s}}
$$

while the variation of $\theta$ is calculated as (cf. equation (5))

$$
\frac{\partial \theta}{\partial p_{s}}=\int_{0}^{t^{F}} A \frac{\Delta E U^{s}}{R T^{2}} \exp \left[-\frac{\Delta E}{R T}\right] \mathrm{d} t
$$


Equation (9) is supplemented by boundary conditions in the form

$$
\mathbf{x} \in \Gamma_{0}: \begin{cases}Q^{s}(\mathbf{x}, t)=-\frac{1}{\lambda} \frac{\partial \lambda}{\partial p_{s}} q(\mathbf{x}, t), & t \leq t_{\exp } \\ Q^{s}(\mathbf{x}, t)=\alpha U^{s}-\frac{1}{\lambda} \frac{\partial \lambda}{\partial p_{s}} q(\mathbf{x}, t), & t>t_{\exp }\end{cases}
$$

where

$$
Q^{s}=-\lambda U_{, i}^{s} n_{i}
$$

and the initial one

$$
t=0: \quad U^{s}=0
$$

The change of temperature due to the changes of the parameters $p_{s}$ can be estimated using the following formula

$$
\Delta T=\sqrt{\sum_{s=1}^{n}\left(\frac{\partial T}{\partial p_{s}} \Delta p_{s}\right)^{2}}
$$

\section{Results of computations}

The basic and also additional problems resulting from the sensitivity analysis have been solved using the $1^{\text {st }}$ scheme of the boundary element method for $2 \mathrm{D}$ transient heat diffusion $[5,10]$.

The domain of rectangular shape (cf. Fig. 1) of dimensions $0.05 \times 0.015 \mathrm{~m}$ is considered. The interior of domain has been divided into 6000 internal constant cells, while the external boundary into 320 constant elements.

In computations, the following values of tissue parameters have been assumed: $\lambda=0.3 \mathrm{Wm}^{-1} \mathrm{~K}^{-1}, c=3.647 \mathrm{MJm}^{-3} \mathrm{~K}^{-1}, G_{B 0}=0.00125\left(\mathrm{~m}_{\text {blood }}^{3} \mathrm{~s}\right) /\left(\mathrm{m}_{\text {tissue }}^{3}\right), Q_{\text {met }}=$ $=245 \mathrm{Wm}^{-3}$, while for the blood $c_{B}=3.9962 \mathrm{MJm}^{-3} \mathrm{~K}^{-1}$ and $T_{B}=37^{\circ} \mathrm{C}$. The parameters of Arrhenius injury integral are: $A=3.1 \cdot 10^{98} \mathrm{~s}^{-1}, E=6.27 \cdot 10^{5} \mathrm{~J} \mathrm{~mole}^{-1}$, $R=8.314 \mathrm{~J} \mathrm{~mole}^{-1} \mathrm{~K}^{-1}$ and $\theta_{\text {rec }}=0.05$, and the complete form of perfusion coefficient definition (cf. equations (3) and (4)) is [4]:

$$
G_{B}(\theta)= \begin{cases}G_{B 0}, & \theta=0 \\ \left(1+25 \theta-260 \theta^{2}\right) G_{B 0}, & 0<\theta \leq 0.1 \\ (1-\theta) G_{B 0}, & 0.1<\theta \leq 1 \\ 0, & \theta>1\end{cases}
$$


The values of coefficients for the interval from 0 to 0.1 respond to the increasing of the perfusion coefficient caused by vasodilatation up to the value $\theta=0.05$ (maximum of the function) and the beginning of the narrowing of blood vessels (between 0.05 and 0.1 ). The interval 0.1 to 1 they reflect blood flow decrease as the vasculature is going to shut down [2].

In the boundary condition (cf. equation (2)) the following values of parameters have been assumed: $\alpha=10 \mathrm{Wm}^{-2} \mathrm{~K}^{-1}$ and $T_{a m b}=20^{\circ} \mathrm{C}$, maximal value of the heat flux $q_{0}$ is assumed as $20 \mathrm{kWm}^{-2}$ while the exposure time is 30 seconds. The time step $\Delta t=1 \mathrm{~s}$.

The co-ordinates of the points are (cf. Fig. 1): $\mathrm{C}_{1}(0.01575,0.001875)$, $\mathrm{D}_{1}(0.03475,0.000625), \mathrm{C}_{2}(0.01575,0.003125)$ and $\mathrm{D}_{2}(0.03475,0.000875)$.

As it was previously mentioned, the sensitivity analysis has been performed with regard to thermal conductivity, volumetric specific heat, initial perfusion coefficient and metabolic heat source. It is assumed that for all parameters $\Delta p_{s}=0.1 p_{s}$.

Figure 3 shows the courses of the temperature as well as the courses of the injury integral $\theta$. At two of these points, $C_{1}$ and $D_{1}$, the value of the injury integral is above the recovery threshold $\theta_{\text {rec }}$. At the point $C_{1}$ the value of the injury integral is much greater than 1 , so the tissue is fully damaged, while at the point $D_{1}$ the value of the injury integral is 0.168 , which means partly damaged tissue. Arrhenius integral values at points $C_{2}$ and $D_{2}$ haven't reached the recovery threshold, so the functions $\theta_{\text {app }}$ are defined for the stage of lowering temperature.
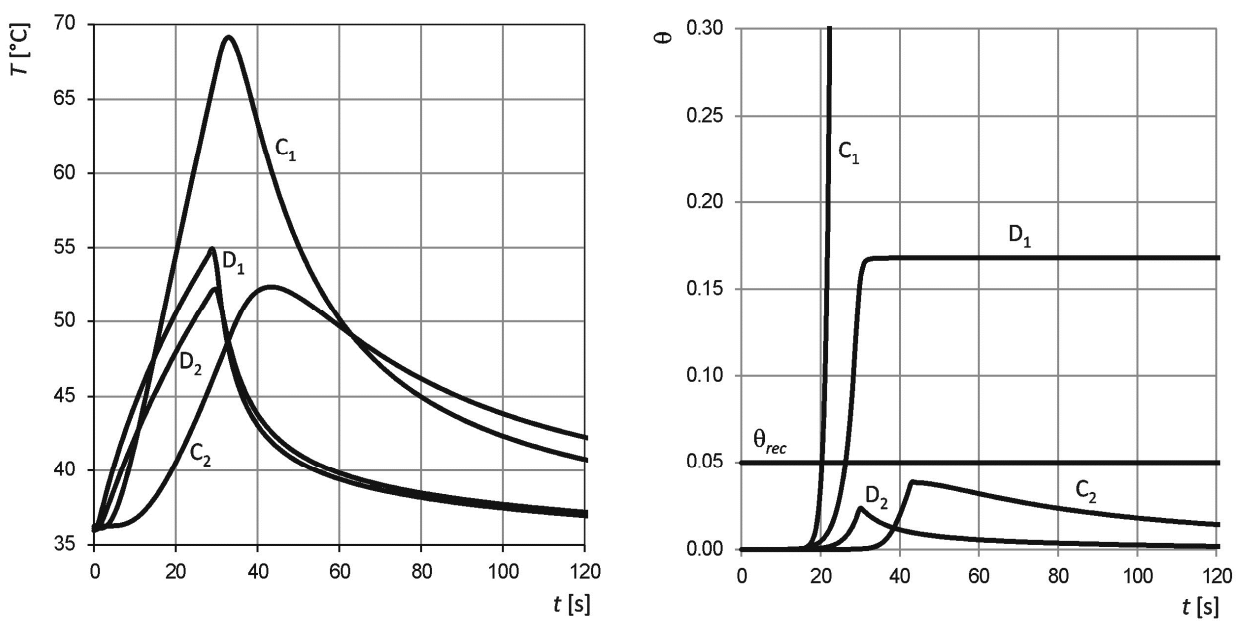

Fig. 3. Courses of temperature and the injury integral $\theta$

The next two figures present the profiles of sensitivity function for successive thermophysical parameters of tissue. It turns out that $10 \%$ changes in parameters $p_{s}$ values have effects mainly in the sensitivity functions of thermal conductivity $\lambda$ 
and volumetric specific heat. The maximal changes are noticed at the points $\mathrm{C}_{1}$ and $\mathrm{C}_{2}$.

The sensitivity function of the initial perfusion coefficient $G_{B 0}$ achieved a value much below $1{ }^{\circ} \mathrm{C}$ (absolute value) while the values of sensitivities for metabolic heat source $Q_{m e t}$ are practically negligible.

It should be pointed out that in each of the four cases, the courses of sensitivity functions at the points $D_{1}$ and $D_{2}$ were very similar.
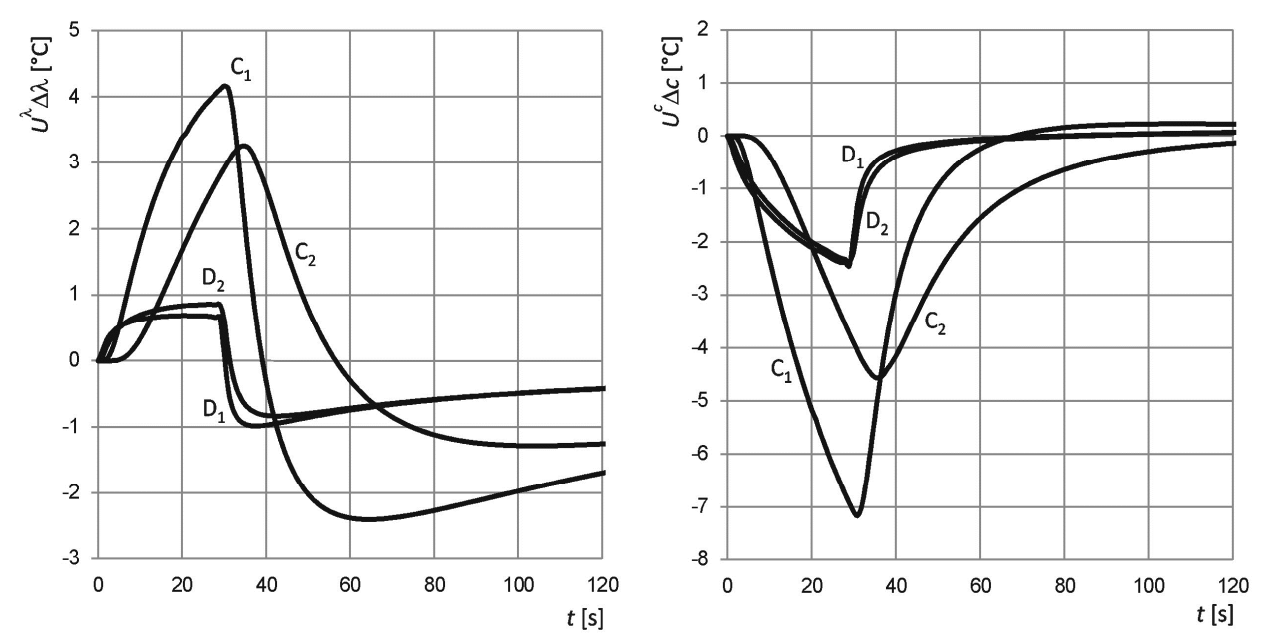

Fig. 4. Courses of sensitivity function of $\lambda$ and $c$
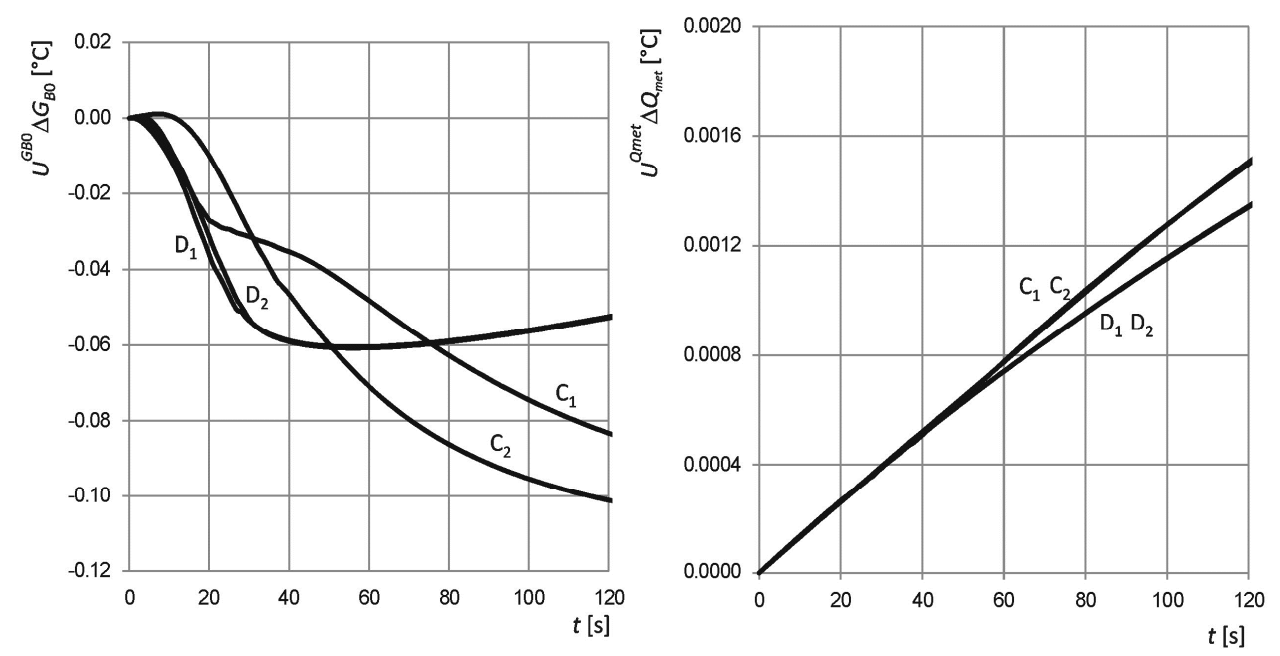

Fig. 5. Courses of sensitivity function of $G_{B 0}$ and $Q_{m e t}$ 
In the Figure 6 the changes of temperature due to the changes of parameters $p_{s}$ are shown (cf. equation (19)). As for the single parameters the maximal changes are noticed at the points $\mathrm{C}_{1}$ and $\mathrm{C}_{2}\left(8.6\right.$ and $5.75^{\circ} \mathrm{C}$ respectively) as well as the courses at the points $\mathrm{D}_{1}$ and $\mathrm{D}_{2}$ are very alike (the changes up to $2.6^{\circ} \mathrm{C}$ ).

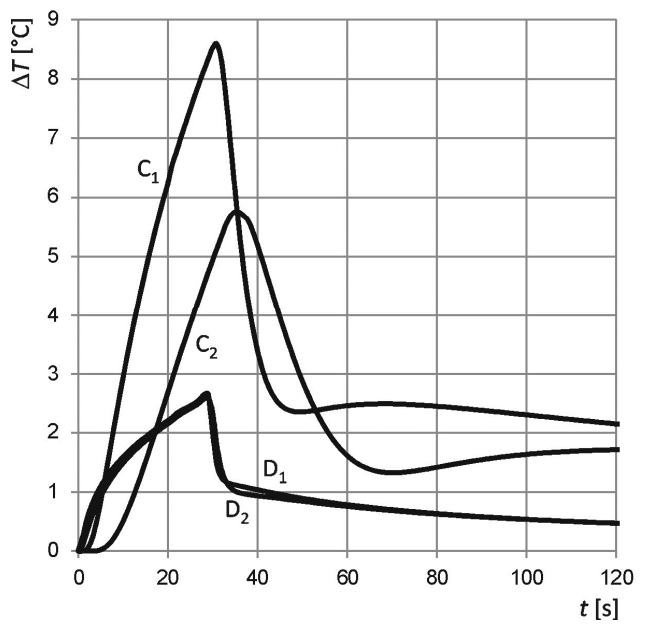

Fig. 6. Changes of temperature due to the changes of parameters $p_{s}$

\section{Final remarks}

The profiles of the sensitivity functions show that thermal conductivity and volumetric specific heat have the most substantial impact on temperature levels. It should be pointed out that for these two parameters maximal values of sensitivity function at the point $C_{1}$ (as well as at the points $D_{1}$ and $D_{2}$ ) are achieved about time when the external heat impulse is ceased while for the point $C_{2}$ the maximal values are reached a few seconds later. This is due to the necrotic zone around the point $\mathrm{C}_{1}$. As it was written previously, the value of tissue injury integral at that point is much greater than 1 , so it could be treated as the area of fully damaged tissue (cf. Fig. 3).

Application of the Arrhenius formulation in such a kind of problems seems to be quite a convenient tool to obtain additional information about the process considered. The proposed method of tissue injury calculation is closer to the real conditions of interaction between tissue and high-temperature impulse. Its main advantage is the possibility of estimation of the tissue recovery area, which could have an important part in the case of modelling of a fully controlled case of interaction, such as prostate hyperplasia or cancer thermotherapy.

It should be pointed out that the presented model could also be considered with phase change taken into account, similarly to that presented in [2]. At the stage of sensitivity analysis using the adjoint approach is also possible. 


\section{References}

[1] Jasiński M., Modelling of 1D bioheat transfer with perfusion coefficient dependent on tissue necrosis, Scientific Research of the Institute of Mathematics and Computer Science 2008, 7, $57-$ -62 .

[2] Abraham J.P., Sparrow E.M., A thermal-ablation bioheat model including liquid-to-vapor phase change, pressure- and necrosis-dependent perfusion, and moisture-dependent properties, Int. Journal of Heat and Mass Transfer 2007, 50, 2537-2544.

[3] Henriques F.C., Studies of thermal injuries, V. The predictability and the significance of thermally induced rate process leading to irreversible epidermal injury, Archives of Pathology 1947, 43, 489-502.

[4] Jasiński M., Investigation of tissue thermal damage process with application of direct sensitivity method, MCB: Molecular \& Cellular Biomechanics 2013, 10, 3, 183-199.

[5] Mochnacki B., Majchrzak E., Sensitivity of the skin tissue on the activity of external heat sources, CMES: Computer Modeling in Engineering and Sciences 2003, 4, 3-4, 431-438.

[6] Jasiński M., Sensitivity analysis of transient bioheat transfer with perfusion rate dependent on tissue injury, Computer Assisted Mechanics and Engineering Science 2009, 16, 267-277.

[7] Dems K., Sensitivity analysis in thermal problems. Part I: Variation of material parameters within fixed domain, Journal of Thermal Stresses 1986, 9, 303-324.

[8] Jasiński M., Sensitivity analysis of bioheat transfer in human cornea subjected to laser irradiation. Part 1: Variation of optical parameters, Scientific Research of the Institute of Mathematics and Computer Science 2009, 8, 63-70.

[9] Kleiber M., Parameter Sensitivity in Nonlinear Mechanics, J.Wiley \& Sons Ltd, Chichester 1997.

[10] Majchrzak E., Application of different variants of the BEM in numerical modeling of bioheat transfer processes, MCB: Molecular \& Cellular Biomechanics 2013, 10, 3, 201-232. 